\title{
EDUCAÇÃO EM SAÚDE E A ATUAÇÃO DE ENFERMAGEM NO CONTEXTO DE UNIDADES DE INTERNAÇÃO HOSPITALAR: O QUE TEM SIDO OU HÁ PARA SER DITO?
}

\author{
Angelita Gastaldo Rigon ${ }^{1}$, Eliane Tastch Neves ${ }^{2}$
}

\footnotetext{
${ }^{1}$ Mestranda do Programa de Pós-Graduação em Enfermagem (PPGEnf) da Universidade Federal de Santa Maria (UFSM). Enfermeira do Hospital Universitário de Santa Maria. Rio Grande do Sul, Brasil. E-mail: enfangel@ibest.com.br

${ }^{2}$ Doutora em Enfermagem. Professora Adjunto do Departamento de Enfermagem e do PPGEnf da UFSM. Rio Grande do Sul, Brasil. E-mail: elianeves03@gmail.com
}

RESUMO: A presente revisão foi fundamentada em uma busca bibliográfica desenvolvida no portal de teses e dissertações da Coordenação de Aperfeiçoamento Pessoal de Nível Superior, em novembro de 2009, com recorte temporal delimitado entre 1998 e 2008 , utilizando-se os termos "educação em saúde", "enfermagem" e "hospital" como assunto. Foram incluídos trabalhos desenvolvidos no âmbito hospitalar e/ou apresentando reflexões sobre o tema. Analisaram-se 22 produções. Foram identificadas dez, referentes à educação em saúde, realizadas por enfermeiros, no contexto de internação hospitalar, com utilização da perspectiva de autonomia, como forma de promoção da saúde e nove foram implementadas e/ ou propostas por pesquisadores e não por enfermeiros assistenciais das unidades de internação. Concluiu-se que há necessidade de maior desenvolvimento do tema educação em saúde, como inerente aos cuidados de enfermagem no contexto da internação hospitalar. Recomenda-se uma abordagem pautada em pedagogias ativas, possibilitando uma prática da enfermagem emancipatória e o empoderamento de sujeitos e enfermeiros.

DESCRITORES: Educação em saúde. Enfermagem. Cuidados de enfermagem. Hospitalização.

\section{HEALTH EDUCATION AND NURSING PRACTICE IN THE HOSPITALIZATION CONTEXT: WHAT HAS BEEN OR IS TO BE SAID?}

\begin{abstract}
This paper was grounded on a bibliographic search in November, 2009, through the Brazilian Government Agency database (Coordenação de Aperfeiçoamento de Pessoal de Nivel Superior) for Master's theses and PhD dissertations. The period under examination was delimited between 1998 and 2008, searching for the terms "health education", "nursing", and "hospital". Studies developed or carried out in the hospital context were included as well as/or theoretical reflections on this theme. Twenty-two papers were analyzed. Ten were identified which referred to health education and were conducted by nurses in the hospital unit context under a perspective of autonomy as a way to promote health. Nine were implemented and/or proposed by researchers who were not nursing caregivers at these units. This study concludes that health education needs to be inserted as inherent to nursing care in the hospital inpatient context. An approach grounded on active pedagogies is recommended in order to make an emancipating nursing practice and the empowerment of subjects and nurses possible.
\end{abstract}

DESCRIPTORS: Health education. Nursing. Nursing care. Hospitalization.

\section{LA EDUCACIÓN EN SALUD Y LA ACTUACIÓN DE LA ENFERMERÍA EN EL CONTEXTO DE LAS UNIDADES DE INTERNACIÓN HOSPITALARIA: ¿QUÉ HA SIDO DICHO Y QUÉ HAY POR DECIR?}

\begin{abstract}
RESUMEN: Esta revisión se basó en una búsqueda bibliográfica desarrollada en el portal de tesis doctorales y de maestría de la Coordenação de Aperfeiçoamento Pessoal de Nível Superior, en noviembre de 2009. Se hizo un recorte temporal comprendido entre los años 1998 y 2008, por medio de los términos: "educación en salud", "enfermería” y “hospital” como tema. Se incluyeron trabajos desarrollados en el contexto hospitalario y/o que presentaban reflexiones acerca del tema. Se analizaron 22 estudios. Se identificaron diez referentes a la educación en salud realizados por enfermeros en un contexto de internación hospitalaria con la utilización de la perspectiva de la autonomía como forma de promoción de la salud. Nueve se han implementado y/o se han propuesto por investigadores y no por enfermeros asistenciales de las unidades. Se concluye que existe la necesidad de un mayor desarrollo del tema de la educación en salud como actividad inherente a los cuidados de enfermería en el contexto de la internación hospitalaria. Se recomienda una abordaje con la utilización de pedagogías activas, que posibiliten una práctica de enfermería de emancipación y autonomía de los sujetos y enfermeros.
\end{abstract} DESCRIPTORES: Educación en salud. Enfermería. Atención de enfermería. Hospitalización. 


\section{INTRODUÇÃO}

As atividades do enfermeiro têm se diversificado e ampliado, tornando-se um processo complexo, sendo compreendido pelo cuidar, educar e gerenciar. Entretanto, tem-se observado que, na prática, alguns enfermeiros possuem uma visão limitada e limitante do cuidado, vendo-o, muitas vezes, de uma maneira compartimentada. Em geral, essa prática volta-se à implementação de cuidados seriados dentro de uma lógica resolutiva, ${ }^{1}$ em que o educar nem sempre está presente. Acredita-se que o cuidar, associado ao educar, possibilita conversão e diversificação dos conhecimentos, onde estes possam ser construídos, desconstruídos e adaptados ${ }^{1}$ às necessidades individuais e coletivas.

Nesse sentido, a educação em saúde perpassa o cotidiano do enfermeiro, considerando a recuperação, prevenção e as necessidades de ensino do paciente. Essa realidade inclui o âmbito hospitalar, onde o enfermeiro é chamado a reconstruir sua prática de cuidado direto, para um modelo mais abrangente, em que a educação faz parte da assistência. Assim, o enfermeiro hospitalar também deve transpor seus conhecimentos "de modo a permitir uma mudança na lógica de atenção, onde o enfoque pode deixar de ser apenas o tratamento da doença, para contemplar uma questão mais abrangente em que o indivíduo, que se encontra momentaneamente doente, após, retornará para o contexto social do qual faz parte" .2: 297

Educar significa um processo baseado na reflexão da realidade, no diálogo e na troca de experiências entre educador/educando e profissional/ cliente possibilitando que ambos aprendam juntos, por meio de processo emancipatório. ${ }^{3}$ Essa ideia vem ao encontro da pedagogia libertadora e problematizadora, em que a troca de conhecimentos ultrapassa o campo específico da educação somente, transformando-a em educação para o mundo e do mundo para educação, numa possibilidade de transformação deste mundo por meio de uma ação consciente. ${ }^{4}$

Dessa forma, o conceito de educação em saúde soma-se ao conceito de promoção da saúde, utilizando a educação como uma forma de cuidar, transcendendo os preceitos básicos do cuidado. Assim, ao educar, potencializamos nossa capacidade de cuidar, e capacitamos intervenções de forma construtivo-reflexiva, singular-plural, dinâmico-flexível, num sistema cíclico de relações interpessoais, dentro de uma realidade histórica cultural, em que um aprende com o outro, e este aprender possibilita a transformação de ambos, de quem os rodeiam e do meio no qual estão inseridos, ${ }^{1}$ transcendendo o tempo em que se realiza o cuidado/educação.

Assim, na experiência profissional das autoras, percebe-se a atuação hospitalar do enfermeiro centrada nos aspectos biológicos e tecnológicos, ocupando-se em funções que se dividem entre as exigências da atuação na assistência e no gerenciamento, encontrando pouco tempo para o desenvolvimento de atividades de educação em saúde.

Diante do exposto, pesquisar o que tem sido produzido concernente ao tema educação em saúde no contexto de internação hospitalar, tornou-se uma inquietação. Com isso, objetivou-se neste artigo descrever e discutir a produção científica no banco de teses e dissertações da Coordenação de Aperfeiçoamento Pessoal de Nível Superior (CAPES), considerando a educação em saúde em unidades de internação hospitalar.

\section{DESENVOLVIMENTO}

A presente revisão foi fundamentada em uma busca bibliográfica desenvolvida no portal de teses e dissertações da CAPES, em novembro de 2009, com recorte temporal delimitado entre 1998 e 2008, utilizando-se os termos educação em saúde, enfermagem e hospital como assunto. Foram encontradas 143 dissertações e 36 teses, totalizando 179 produções. Procedeu-se a leitura dos títulos destas para a seleção. Utilizou-se, como critério de inclusão, o fato de os trabalhos serem desenvolvidos no âmbito hospitalar ou apresentarem reflexões sobre o tema, e, como critérios de exclusão, os trabalhos desenvolvidos fora do âmbito hospitalar e/ou que não incluíssem pacientes hospitalizados ou enfermeiros da assistência hospitalar.

Foram selecionadas 30 dissertações e nove teses, totalizando 39 produções para análise, por meio da leitura dos resumos. Para a organização e a análise dos dados, foi utilizado um quadro sinóptico, contendo as seguintes informações: autor(es), cenário(s), sujeitos participantes do estudo, ano de publicação, local do curso de pós-graduação, tipo de estudo, objetivo(s) e conclusão da pesquisa. Após a pré-análise, foram excluídos 17 trabalhos, por não convergirem com o objeto de estudo ou se enquadrarem em algum dos critérios de exclusão, permanecendo 22 trabalhos para a análise e a interpretação. Os dados organizados neste quadro foram submetidos à análise de conteúdo temática. 
O quadro 1 apresenta a lista das produções analisadas, o título, os seus autores, o ano da publicação e a categoria do estudo, ou seja, se dissertação (D) ou tese (T).

\section{Quadro 1 - Teses e Dissertações sobre Educação em Saúde em unidades de internação hospitalar - período 1998-2008 - Portal CAPES}

\begin{tabular}{|c|c|c|c|}
\hline Título & Autor & Ano & Categoria \\
\hline $\begin{array}{l}\text { Educação para a saúde na assistência de enfermagem - um estudo } \\
\text { exploratório em unidade de internação }\end{array}$ & Carlos Bezerra de Lima & 1990 & D \\
\hline $\begin{array}{l}\text { Autocuidado na utilização de medicamentos cardiovasculares: } \\
\text { proposta educativa de enfermagem }\end{array}$ & $\begin{array}{l}\text { Cleide Rejane Damaso } \\
\text { de Araújo }\end{array}$ & 1996 & $\mathrm{D}$ \\
\hline $\begin{array}{l}\text { A conscientização como fundamento da educação em saúde às } \\
\text { pessoas hospitalizadas com tuberculose pulmonar }\end{array}$ & $\begin{array}{l}\text { Lita Miriam Moore } \\
\text { Espinoza }\end{array}$ & 1998 & D \\
\hline $\begin{array}{l}\text { Ações educativas da equipe de enfermagem para a promoção da } \\
\text { saúde da criança hospitalizada }\end{array}$ & Aldalice Brait Lima Alves & 2000 & $\mathrm{D}$ \\
\hline $\begin{array}{l}\text { Percepções de uma equipe de enfermagem sobre permanência } \\
\text { conjunta: implicações educacionais }\end{array}$ & Josiane Dalle Mulle & 2000 & $\mathrm{D}$ \\
\hline $\begin{array}{l}\text { O idoso hospitalizado e seu familiar acompanhante: uma proposta } \\
\text { de assistência de enfermagem para o autocuidado por meio da } \\
\text { educação participativa }\end{array}$ & Jordelina Schier & 2001 & $\mathrm{D}$ \\
\hline $\begin{array}{l}\text { Cuidados com o bebê prematuro: cartilha educativa para orientação } \\
\text { materna }\end{array}$ & $\begin{array}{l}\text { Luciana Mara Monti } \\
\text { Fonseca }\end{array}$ & 2002 & $\mathrm{D}$ \\
\hline $\begin{array}{l}\text { A compreensão do ato educativo pela equipe de enfermagem no } \\
\text { contexto hospitalar }\end{array}$ & Rosmari Deggerone & 2003 & $\mathrm{D}$ \\
\hline $\begin{array}{l}\text { Grupos operativos: uma estratégia para a assistência de enfermagem } \\
\text { em uma unidade hospitalar de referência em câncer de mama }\end{array}$ & Fabiana Verdan Simões & 2004 & $\mathrm{D}$ \\
\hline $\begin{array}{l}\text { A enfermagem promovendo a saúde no cuidado a pessoas que } \\
\text { vivenciam cirurgia cardíaca }\end{array}$ & Patricia de Gasperi & 2005 & $\mathrm{D}$ \\
\hline $\begin{array}{l}\text { Promovendo caminhos para a educação em saúde com idosos } \\
\text { portadores de cardiopatias e seus familiares no setor de emergência } \\
\text { do hospital universitário }\end{array}$ & $\begin{array}{l}\text { Penélope Paloma } \\
\text { Rüdiger Scheidt }\end{array}$ & 2006 & $\mathrm{D}$ \\
\hline $\begin{array}{l}\text { O distanciamento da relação enfermeiro - paciente: o cuidado e sua } \\
\text { dimensão educativa em análise }\end{array}$ & Janize Carlos da Silva & 2006 & $\mathrm{D}$ \\
\hline $\begin{array}{l}\text { O processo de cuidar em enfermagem ao portador de doença crônica } \\
\text { cardíaca }\end{array}$ & $\begin{array}{l}\text { Anice de Fátima Ahmad } \\
\text { Balduino }\end{array}$ & 2007 & D \\
\hline $\begin{array}{l}\text { Práticas educativas em saúde com a família da criança hospitalizada: } \\
\text { componente essencial do cuidado de enfermagem }\end{array}$ & $\begin{array}{l}\text { Fernanda Garcia } \\
\text { Bezerra Goés }\end{array}$ & 2007 & $\mathrm{D}$ \\
\hline $\begin{array}{l}\text { Educação em saúde para familiares cuidadores de pacientes } \\
\text { disfágicos pós-acidente vascular cerebral }\end{array}$ & $\begin{array}{l}\text { Valdete Alves da Silva } \\
\text { de Quadros }\end{array}$ & 2007 & D \\
\hline $\begin{array}{l}\text { Competências para ação educativa da enfermeira: uma interface } \\
\text { entre o ensino e a assistência de enfermagem }\end{array}$ & Valeria Marli Leonello & 2007 & $\mathrm{D}$ \\
\hline $\begin{array}{l}\text { Promoção de saúde na atenção terciária: a atuação do profissional de } \\
\text { enfermagem em um hospital de Uberaba - MG }\end{array}$ & Marlúcio Anselmo Alves & 2008 & $\mathrm{D}$ \\
\hline $\begin{array}{l}\text { Construção coletiva de um espaço dialógico com os cuidadores } \\
\text { familiares dos idosos hospitalizados visando a uma educação em } \\
\text { saúde }\end{array}$ & $\begin{array}{l}\text { Tatiana Martins da } \\
\text { Silveira Aragão }\end{array}$ & 2008 & $\mathrm{D}$ \\
\hline $\begin{array}{l}\text { Orientação sistematizada a pacientes internados com doença } \\
\text { transmissível }\end{array}$ & Adelia Maya Chida & 1990 & $\mathrm{~T}$ \\
\hline $\begin{array}{l}\text { A práxis educativa de enfermagem no cuidado hospitalar: discursos } \\
\text { de enfermeiras }\end{array}$ & $\begin{array}{l}\text { Lita Miriam Moore } \\
\text { Espinoza }\end{array}$ & 2007 & $\mathrm{~T}$ \\
\hline $\begin{array}{l}\text { A qualidade da assistência do enfermeiro em unidades pediátricas de } \\
\text { um hospital de ensino: implantação de um programa educativo }\end{array}$ & $\begin{array}{l}\text { Maria Rita Rodrigues } \\
\text { Vieira }\end{array}$ & 2007 & $\mathrm{~T}$ \\
\hline $\begin{array}{l}\text { Gestão do trabalho e educação em saúde: percepção dos } \\
\text { profissionais de saúde }\end{array}$ & $\begin{array}{l}\text { Volnei Gonçalves } \\
\text { Pedroso }\end{array}$ & 2008 & $\mathrm{~T}$ \\
\hline
\end{tabular}




\section{RESULTADOS E DISCUSSÃO}

Dos trabalhos selecionados, 14 foram desenvolvidos em programas de pós-graduação em enfermagem, sendo que, do total de trabalhos selecionados do banco de teses e dissertações da CAPES (22), sete foram desenvolvidos no Estado de São Paulo, e cinco, em Santa Catarina.

Conforme a análise das produções, o tema educação em saúde, no contexto de internação hospitalar, com e por enfermeiros, tem suas primeiras produções, em nível de programas de mestrado e doutorado, publicadas no banco de teses e dissertações da CAPES, a partir de 1990, sendo que a primeira dissertação encontrada objetivava identificar a percepção e expectativas dos clientes internados, a percepção e a prática dos enfermeiros de unidades hospitalares sobre a educação em saúde desses clientes. ${ }^{5}$

Ainda, na década de 1990, foram encontradas mais duas dissertações direcionadas para áreas específicas: pacientes internados com distúrbio cardiovascular e pacientes internados com tuberculose pulmonar. Assim, em uma das muitas formas de conceber a educação em saúde, esta pode servir para sanar a falta de informação que poderia gerar uma interpretação deficiente do tratamento prescrito e dos cuidados de reabilitação, consequentemente, promovendo um tratamento ineficaz. ${ }^{3}$

Portanto, na década de 1990, percebe-se o despontar de trabalhos envolvendo educação em saúde, como forma eficiente de incluir o paciente internado em seu próprio tratamento, num movimento de sinergismo de atitudes e ações.

Salienta-se que este tema foi encontrado, como tese de doutorado, no ano de 1990, sendo que a próxima tese somente foi encontrada em 2007. Desse modo, questiona-se se este tema não tem sido explorado em nível de doutorado, ou se as palavras utilizadas na busca não convergiram com as escolhidas pelos autores dos trabalhos.

No ano de 2000, não foram encontradas produções referentes ao tema. Já, em 2001, uma dissertação, sob a forma de pesquisa convergente-assistencial, desenvolveu uma prática baseada no autocuidado, com ênfase em ação educativa participativa grupal freireana, junto a pacientes idosos hospitalizados. Verificou-se que isso é possível no ambiente hospitalar e que promove a autonomia, vislumbrando uma forma renovada de prática de enfermagem. ${ }^{6}$

Entre os anos de 2004 a 2006, foram encontradas somente dissertações (quatro), destas, destaca- -se uma, de 20057, que desenvolveu uma prática cuidativa-educativa para promoção da saúde de pessoas que se submeteram à cirurgia cardíaca e seus familiares. Nesse trabalho, a pesquisadora enfatiza que o processo de cuidar/educar realizado gerou frutos gratificantes, dentre os quais, a promoção da saúde, serenidade e participação consciente dos pacientes.

A produção de $2006^{8}$ buscou identificar a dimensão educativa, nos momentos de cuidado de enfermeiros, com ênfase no âmbito hospitalar, analisando o distanciamento histórico do enfermeiro do paciente a ser cuidado, sobretudo, na dimensão educativa. Discutiu a reconstrução da identidade do enfermeiro, enquanto educador, intervindo na realidade dos pacientes, auxiliando a transformação, a autonomia e a emancipação.

A concepção do emprego de pedagogias ativas na educação em saúde envolve problematização, em que ensinar é oferecer oportunidades significativas que despertem no educando suas potencialidades e capacidades de reintervenção na realidade, valorizando as experiências de ambos num processo de construção coletiva do conhecimento. ${ }^{9}$

Nesse sentido, a partir de 2006, identificou-se uma ampliação do que seja o desenvolvimento da educação em saúde, abrindo espaço para a utilização de pedagogias ativas que promovam a autonomia. Diferentemente da segunda metade da década de 1990, em que as produções, que envolviam pacientes internados e atividades de educação em saúde, eram desenvolvidas em momentos específicos e direcionadas, majoritariamente, a orientações específicas.

Ainda assim, no ano de 2007, quatro dissertações e duas teses foram selecionadas e, destas, uma abordou a educação em saúde pautada na transmissão de conhecimentos e no modelo biologicista. ${ }^{10}$ Outro estudo, concluiu que o processo educativo é eficaz na melhora do conhecimento dos cuidadores que assumirão o paciente após a alta, preconizando aulas teóricas e orientações à beira do leito num sentido ainda verticalizado de transmissão de informações. ${ }^{11}$

Também no ano de 2007 foi selecionada uma tese que converge com o que se acredita ser o caminho na práxis da enfermagem hospitalar, pois objetivou compreender e analisar a consciência da práxis educativa das enfermeiras e analisar as razões que impedem o desenvolvimento das ações educativas das enfermeiras no cuidado hospitalar. Essa tese contribuiu, concluindo que é passível o 
modo de pensar a educação como um elemento inerente e indissociável no cuidado hospitalar da enfermeira, bem como sua contribuição para uma melhor qualidade no atendimento ao cliente nos serviços de internação e num processo de viver mais saudável. ${ }^{12} \mathrm{Tal}$ constatação vem ao encontro das atuais formas de entender a educação em saúde preconizada pelas políticas de saúde vigentes.

Em 2008, foram encontradas duas dissertações e uma tese. Uma das dissertações realizou um estudo descritivo, com observação participante de como o enfermeiro da atenção terciária tem desenvolvido sua prática educativa, e o tempo empregado nesta prática em um hospital universitário. Os resultados obtidos demonstraram que o enfermeiro tem utilizado pouco tempo da sua atuação diária com ações educativas ao paciente e família, e que esta prática tem seguido uma linha tecnicista e prescritiva em que as atividades de ordem técnica e administrativa são priorizadas. Nesse sentido, a prática educativa do enfermeiro com o paciente e família está focalizada na admissão e alta na unidade de internação e, raramente, na consulta de enfermagem e nas informações e orientações do estado de saúde ao paciente e sua família. ${ }^{13}$

Frente ao descrito acima, percebe-se que as produções que enfocam a educação em saúde realizada por enfermeiros, no contexto de internação hospitalar, ainda são poucas, comparando-se com o número de produções totais. Em especial, no que se refere ao emprego de pedagogias ativas, sob a perspectiva de promover a autonomia, como forma de promoção da saúde, pois apenas sete dissertações e três teses citaram metodologia participativa e/ou reflexiva e/ou conscientizadora e/ou Paulo Freire.

Entende-se, segundo a tendência freireana, e as políticas de saúde vigentes, que não se pode estimular uma pessoa à mudança sem entender sua existência, sua visão e interpretação da realidade em que vive. As mudanças de atitudes causadas por uma educação em saúde seriam consequência do entendimento e da compreensão dessa realidade e não por mero comando externo.

Com isso, destacam-se: oito trabalhos identificaram ou desenvolveram educação em saúde de forma tradicional, mantendo a verticalidade e a hegemonia do conhecimento científico; nove, foram desenvolvidos ou implantaram ações, com metodologia participativa/conscientizadora e/ou buscaram promover a autonomia dos sujeitos, a partir dos pesquisadores, e não, dos profissionais dos serviços pesquisados.
Assim, a tendência da forma tradicional de transmissão do conhecimento identificada na análise das produções ainda está fortemente presente. Destaca-se, ainda, a invisibilidade da prática de educação em saúde pelo enfermeiro no contexto hospitalar, principalmente, com a utilização de metodologias ativas. Isso corrobora o encontrado na literatura científica nacional, que, na atualidade, grande parte dos profissionais de saúde bem intencionados, ainda, trabalha com o conceito de que o usuário/cliente não sabe ou não possui o conhecimento necessário, sendo então transmitido o que lhe falta. ${ }^{14}$

Em termos de política e gestão, no contexto brasileiro, a educação em saúde vem sendo apontada como uma estratégia que visa à promoção da saúde, sensibilizando os indivíduos para o autocuidado e, principalmente, desenvolvendo a capacidade da autonomia dos sujeitos para que possam, assim, decidir o curso de suas vidas. ${ }^{15}$

Nessa perspectiva é que as políticas públicas de saúde vêm sendo alicerçadas, porém possuem implantação, ainda lenta, devido a diversos fatores, o que repercute na prática diária. Entretanto, acredita-se ser possível e eficaz a educação em saúde, sob a perspectiva de promover a autonomia, como forma de promoção da saúde de forma inerente/intrínseca ao cuidado no âmbito da internação hospitalar.

\section{CONCLUSÃO}

É necessário pensar a prática educativa, como inerente e indissociável ao cuidado hospitalar, numa perspectiva de ação-reflexão-ação dialógica e conscientizadora, revendo-se a identidade do enfermeiro, enquanto educador, auxiliando na transformação, autonomia e emancipação dos indivíduos. Acredita-se ser esta práxis uma das formas de valorizar a profissão de enfermagem e ampliar o espaço de atuação profissional.

Portanto, o paradigma da educação em saúde biologicista, centrado no corpo físico e como prescrição de comportamentos, ainda presente, necessita ser suplantado. Com isso, a atividade do cuidar/educar na enfermagem hospitalar precisa ser desenvolvida pautada na perspectiva de uma educação para a transformação, em busca da autonomia do ser humano, visando à transitividade de consciência, de ingênua para crítica, em que o indivíduo é visto e sentido em sua complexidade.

Acredita-se que a educação em saúde desenvolvida em nível de internação hospitalar é 
uma tendência emergente na enfermagem, porém necessita ser estudada, criada e recriada, promovendo interfaces entre o ensino, a pesquisa, a assistência e a gerência em um movimento dialético de construção do conhecimento e de desenvolvimento da ciência da enfermagem.

Assim, conclui-se que a educação em saúde deve ser abordada como inerente aos cuidados de enfermagem, no contexto da internação hospitalar, principalmente, com a utilização de pedagogias ativas, pois as produções encontradas demonstraram a possibilidade de a academia fomentar essa reflexão entre os profissionais.

Recomenda-se que a abordagem participativa seja amplamente empregada, considerando-se que a mesma possa ser o "estopim" para uma discussão dessa temática entre os enfermeiros de unidades de internação e que estes possam agir-refletir-agir sobre sua prática, possibilitando uma prática de enfermagem emancipatória, na perspectiva do empoderamento dos sujeitos e dos enfermeiros.

\section{REFERÊNCIAS}

1. Ferraz F, Silva LWS, Silva LAA, Reibnitz KS, Backes VMS. Cuidar-educando em enfermagem: passaporte para o aprender/educar/cuidar em saúde. Rev Bras Enferm. 2005 Set-Out; 58(5):607-10.

2. Lucena AF, Paskulin LMG, Souza MF, Gutiérrez MGR. Construção do conhecimento e do fazer enfermagem e os modelos assistenciais. Rev. Esc. Enferm USP. 2006 Jun; 40(2):292-8.

3. Vila ACD, Vila VSC. Tendências da produção do conhecimento na educação em saúde e no Brasil. Rev. Latino-am. Enferm. 2007 Nov-Dez; 15(6):1177-83.

4. Freire P. Pedagogia do oprimido. $17^{\mathrm{a}}$ ed. Rio de Janeiro: Paz e Terra; 1987.

5. Lima CB. Educação para a saúde na assistência de enfermagem - um estudo exploratório em unidade de internação [dissertação]. Rio de Janeiro (RJ): Universidade Federal do Rio de Janeiro, Programa de Pós-Graduação em Enfermagem; 1990.

6. Schier J. O idoso hospitalizado e seu familiar acompanhante: uma proposta de assistência de enfermagem para o autocuidado por meio da educação participativa. [dissertação]. Florianópolis (SC): Universidade Federal de Santa Catarina, Programa de Pós-Graduação em Enfermagem; 2001.

7. Gasperi P. A enfermagem promovendo a saúde no cuidado a pessoas que vivenciam cirurgia cardíaca [dissertação]. Florianópolis (SC): Universidade Federal de Santa Catarina, Programa de PósGraduação em Enfermagem; 2005.

8. Silva JC. O distanciamento da relação enfermeiropaciente: o cuidado e sua dimensão educativa em análise [dissertação]. São Paulo (SP): Universidade Metodista de São Paulo, Programa de Pós-Graduação em Educação; 2006.

9. Reibnitz KS, Prado ML. Em busca de metodologias ativas: possibilidades e desafios da metodologia problematizadora. In: Reibnitz KS, Prado ML. Inovação e educação em enfermagem. Florianópolis (SC): Cidade Futura; 2006. p. 223-39.

10. Góes FGB. Práticas educativas em saúde com a família da criança hospitalizada: componente essencial do cuidado de enfermagem [dissertação]. Rio de Janeiro (RJ): Universidade Federal do Rio de Janeiro, Programa de Pós-Graduação em Enfermagem; 2007.

11. Quadros VAS. Educação em saúde para familiares cuidadores de pacientes disfágicos pós-acidente vascular cerebral [dissertação]. Curitiba (PR): Universidade Tuiuti, Programa de Pós-Graduação em Enfermagem - Distúrbios da Comunicação; 2007.

12. Espinoza LMM. A práxis educativa de enfermagem no cuidado hospitalar: discursos de enfermeiras. [tese]. Florianópolis (SC): Universidade Federal de Santa Catarina, Programa de Pós-Graduação em Enfermagem; 2007.

13. Alves MA. Promoção de saúde na atenção terciária: a atuação do profissional de enfermagem em um hospital de Uberaba (MG) [dissertação]. Franca (SP): Universidade de Franca, Curso de Pós-Graduação em Enfermagem; 2008.

14. Boehs AE, Monticelli M, Wosny AM, Heidemann IBS, Grisotti M. A interface necessária entre enfermagem, educação em saúde e o conceito de cultura. Texto Contexto Enferm. 2007 Abr-Jun; 16(2):307-14.

15. Dall' Agnol CM, Resta DG, Zanatta E, Schrank G, Maffacciolli R. O trabalho com grupos como instância de aprendizagem em saúde. Rev Gaúcha Enferm. 2007 Mar; 28(1):21-6. 